\title{
Postoperative Respiratory Complications of Laryngeal Mask Airway and Tracheal Tube in Ear, Nose and Throat Operations
}

\author{
Reza Safaeian ${ }^{1, *}$; Valiollah Hassani ${ }^{1}$; Gholamreza Movasaghi ${ }^{2}$; Mahzad Alimian ${ }^{1}$; Hamid \\ Reza Faiz ${ }^{1}$ \\ ${ }^{1}$ Department of Anesthesiology, Rasoul Akram Medical Center, Iran University of Medical Scienses, Tehran, Iran \\ ${ }^{2}$ Department of Anesthesiology, Hasheminejad Hospital, Iran University of Medical Scienses, Tehran, Iran \\ *Corresponding author: Reza Safaeian, Department of Anesthesiology, Rasoul Akram Medical Center, Iran University of Medical Scienses, Tehran, Iran. Tel: +98-9121437850, Fax: \\ +90-2166509059, E-mail: rezasafaa@hotmail.com
}

Received: November 27, 2014; Revised: March 3, 2015; Accepted: April 18, 2015

\begin{abstract}
Background:Supraglottic devices could be used to reduce postoperative respiratory complications, but there are few studies focused on their use in more prolonged surgeries.

Objectives: In this study, we compared postoperative respiratory complications in patients with prolonged ear, nose and throat (ENT) surgeries, whose airways were controlled with tracheal tube or laryngeal mask airway (LMA).

Materials and Methods: In a randomized control trial (RCT), 171 candidates of prolonged ENT surgeries were randomly assigned into two groups. In group one $(n=85)$ LMA and in group two $(n=86)$ endotracheal tube were used for airway control. The incidences of four postoperative respiratory complications including sore throat, hoarseness, cough and shortness of breath in immediate postoperative period were measured and compared among patients of each group.

Results: Sore throat was recorded in $32.9 \%$ of patients with LMA and $44.2 \%$ of intubated patients, but it was not statistically significant (Fisher's Exact test $=0.158$ ). Hoarseness was recorded in 3.5\% of patients with LMA and $24.4 \%$ of intubated patients (Fisher's Exact test $=0.000$ ). In $1.2 \%$ of patients with LMA cough was recorded; it was also seen in $7 \%$ of the intubated patients (Fisher's Exact test $=0.005$ ). Shortness of breath was mentioned by two intubated patients (2.3\%) and in patient with LMA we did not record this complication.

Conclusions: LMA in prolonged ENT surgeries was associated with reduced respiratory complications.
\end{abstract}

Keywords: Laryngeal Mask Airway; Endotracheal Intubation; Hoarseness

\section{Background}

Postoperative respiratory complications are common in surgical patients. Trauma of airway maneuvers (laryngoscopy) and devices (tracheal tube) are among the different mechanisms involved in these incidences. These complications could be more serious in prolonged surgeries. A suitable airway control device must protect airways from aspiration. In elective surgeries, the risk of gastric content aspiration of laryngeal mask airway (LMA) is not more than tracheal tube. In addition, these devices that have no access to the trachea do not traumatize the region and there are too many studies focused on reducing postoperative respiratory complications with supraglottic devices.

There is a general tendency towards using supraglottic devices in ear, nose and throat (ENT) surgeries (1). Unlike many surgeries, there is no need for high intraoperative inspiratory pressure. Although the patient's head is out of reach, it is usually fixed during the whole length of the surgery. Laryngeal mask can effectively protect lower airways against blood, secretions and irrigation fluids during surgery. Even in prolonged ENT surgeries, supraglot- tic devices are good choices and the length of surgery is not a definite limiting factor for their use.

\section{Objectives}

In this study, patients with prolonged ENT surgeries who were more prone to respiratory complications (2) were selected and the incidences of four respiratory complications including sore throat, hoarseness, cough and shortness of breath in immediate postoperative period were compared between laryngeal mask and endotracheal tube. These symptoms and signs are sources of postoperative discomfort in surgical patients. In addition, they could be surrogate markers of the respiratory system injury and indicate the parts of respiratory system that are more prone to be insulted by airway devices.

\section{Materials and Methods}

In a double-blind randomized control study, 171 candidates of the American society of anesthesiologists physical status classes I and II for ENT surgeries of more than

Copyright (C) 2015, Iranian Society of Regional Anesthesia and Pain Medicine(ISRAPM). This is an open-access article distributed under the terms of the Creative Commons Attribution-NonCommercial 4.0 International License (http://creativecommons.org/licenses/by-nc/4.0/) which permits copy and redistribute the material just in noncommercial usages, provided the original work is properly cited. 
90 minutes were enrolled in Hazrat-e-Rasol medical complex (Tehran, Iran). Approval was obtained from a local ethics committee (IRB) and all the human subjects signed written informed consents. The study protocol confirmed the ethical guidelines of the 1975 Declaration of Helsinki. Although we did not want to have any age limitation, we chose patients to whom we could have a clear communication. The ages of the patients were more than 13 years; they must have had no airway anomalies, history of chronic or acute respiratory diseases or smoking. Obese patients, pregnant women, and patients with gastroesophageal reflux, drug addiction or chronic use of corticosteroid drugs were excluded from the study. Most of the ENT surgeries in this study were ear surgeries, functional endoscopic sinus surgery (FESS), rhinoplasty, parotid surgeries and septoplasty. Patients with surgeries on throat and lower regions of the respiratory system were excluded from the study.

Patients were randomly (table of random numbers) divided into two groups. In the first group LMA and in the second group endotracheal tube were used for airway control. In both groups single-use polyvinyl chloride (PVC) devices were used. The size of LMA for each patient was the recommended size based on the ideal weight of the patient and for endotracheal tube it was the estimated size of the glottis during laryngoscopy. The volume of air for inflating cuffs in both devices was according to the Just-Seal volume method and was as low as just preventing leakage during normal ventilation. Classic method was used for placement of LMA and K-Y jelly was used for lubrication. We used mouth pack for all the patients.

Fentanyl, $5 \mu \mathrm{g} / \mathrm{kg}$ was used for narcotic loading dose. Propofol, $2 \mathrm{mg} / \mathrm{kg}$ and atracurium, $0.5 \mathrm{mg} / \mathrm{kg}$ were used for induction and muscle relaxation, respectively. After tracheal intubation (laryngoscopy) or LMA insertion (classic method), anesthesia was maintained with propofol, $100 \mu \mathrm{g} / \mathrm{kg} / \mathrm{min}$ and fentanyl, $3 \mu \mathrm{g} / \mathrm{kg} / \mathrm{h}$. Standard monitoring was used during the anesthesia and $85 \% \mathrm{O}_{2}, 4$ $\mathrm{L} / \mathrm{min}$ was used for ventilation. At the end of the surgery, the airway device was withdrawn when the patient was fully awake. Postoperative pain control protocol of the ENT ward was applied for all of the patients. One to two hours after discharging the patients from the recovery room we asked them about four postoperative respiratory complications: sore throat, hoarseness, cough and shortness of breath. Positive or negative responses were recorded by independent observers who were not aware of the airway control method in each patient.

According to the literature, $45 \%$ of intubated patients develop sore throat following general anesthesia. We hypothesized that by using laryngeal mask this number should drop to $20 \%$. Therefore, with an alpha error equal to 0.05 and $90 \%$ power, we needed 155 subjects in two equal groups to examine this in a two-sided test approach; adding 10\% for possible dropouts, we needed about 171 patients.

We compared the proportions using chi-squared test.
T-test was used for comparing the means. IBM SPSS statistics version 19.0, Armonk, NY, IBM Corp. was used for data analyses.

\section{Results}

Sixty-eight patients were male and 103 were female. Laryngeal mask was used in $41 \%$ of male patients and 55\% of female patients. The range of patients' age was $13-83$ years. The lengths of surgeries were from 90 minutes to five hours and 45 minutes. We divided the patients according to the length of their surgery into three groups:

1. Operation time $=90-149$ minutes.

2. Operation time $=150-209$ minutes.

3. Operation time $\geq 210$ minutes.

Table 1 indicates the baseline comparison of the patients and randomizations that seemed to be properly performed.

We considered sore throat as any kind of unpleasant sensation in the throat region like pain with or without swallowing. Sore throat was recorded in $32.9 \%$ of patients with LMA and $44.2 \%$ of the intubated patients, but it was not statistically significant (Fisher's exact test $=0.158$ ). Hoarseness was recorded in $3.5 \%$ of patients with LMA and $24.4 \%$ of the intubated patients (Fisher's exact test $=$ $0.000)$. In $1.2 \%$ of patients with LMA cough was reported; it was also seen in $12.8 \%$ of intubated patients (Fisher's exact test $=0.005)$. Shortness of breath was mentioned by two intubated patients (2.3\%) and in the LMA group we did not record this complication. We compared the number of patients with one and more than one complaint between the two groups. Table 2 shows the number of patients with at least one complication and patients who had more than one complication and both of these figures were greater in the intubated patients.

We asked the patients to describe their recorded severity of sore throat, which was described by patients as mild, moderate and severe and there was no significant difference in the severity of sore throat between the two groups. We divided our patients into five age groups: less than 15, 15 - 29, 30 - 44, 45 - 59 and more than 60 years old. The highest incidence of sore throat was recorded in 15 - 29-year-old patients and it decreased in older groups. Higher incidence of cough was recorded in female patients (10.7\% versus $1.5 \%, \mathrm{P}=0.029)$.

Among these respiratory complications, the incidence of hoarseness had a significant relationship with the duration of operation in the intubated patients (Figure 1) and it was more common in intubated patients whose surgeries lasted more than 210 minutes.

Three patients in the LMA group were exited from the study because we changed their LMA to tracheal tube. In one of these patient LMA could not maintain adequate ventilation before the start of surgery. In the other two, although LMA effectively ventilated the patient, the fog produced by minimal leakage of air and the blood splash obscured the endoscopy lens and we had to change LMA to tracheal tube. 


\begin{tabular}{|c|c|c|c|}
\hline & $\begin{array}{c}\text { Intervention Group (Laryn- } \\
\text { geal Mask Airway) }\end{array}$ & Control Group (Tracheal Tube) & PValue \\
\hline Gender & & & $\chi^{2}=3.29^{a}$ \\
\hline Male & $28(32.9 \%)$ & $40(39.8 \%)$ & \\
\hline Female & $57(67.1 \%)$ & $46(53.5 \%)$ & \\
\hline Age Group, y & & & $\chi^{2}=0.90$ \\
\hline$<30$ & $41(48.2 \%)$ & $38(44.2 \%)$ & \\
\hline $30-44$ & $21(24.7 \%)$ & $29(33.7 \%)$ & \\
\hline $45-59$ & $13(15.3 \%)$ & $13(15.1 \%)$ & \\
\hline$>60$ & $10(11.8 \%)$ & $6(7.0 \%)$ & \\
\hline Duration of Operation, min & & & $\chi^{2}=0.55$ \\
\hline $90-149$ & $32(37.6 \%)$ & $30(34.9 \%)$ & \\
\hline $150-209$ & $29(34.1 \%)$ & $36(41.9 \%)$ & \\
\hline$>210$ & $24(28.2 \%)$ & $20(23.3 \%)$ & \\
\hline \multicolumn{4}{|l|}{${ }^{\mathrm{a}} \chi^{2}$ : Chi-squared. } \\
\hline Complications & $\begin{array}{c}\text { Intervention Group } \\
\text { (Laryngeal Mask Airway) }\end{array}$ & Control Group (Tracheal Tube) & PValue \\
\hline Cough & $1(1.2 \%)$ & $11(12.8 \%)$ & $\mathrm{P}=0.005$ \\
\hline Sore throat & $28(32.9 \%)$ & $38(44.2 \%)$ & $P=0.158$ \\
\hline Shortness of breath & $0(0.0 \%)$ & $2(2.3 \%)$ & Fisher's Exact $\mathrm{P}=0.251$ \\
\hline Hoarseness & $3(3.5 \%)$ & $21(24.4 \%)$ & $\mathrm{P}<0.001$ \\
\hline Total No. of complications & & & $\mathrm{P}<0.001$ \\
\hline One complication & $29(34.1 \%)$ & $32(37.2 \%)$ & \\
\hline Two or more & $2(2.4 \%)$ & 19 (22.1\%) & \\
\hline
\end{tabular}

Figure 1. The Incidence of Immediate Postoperative Hoarseness in Patients With Endotracheal Tube

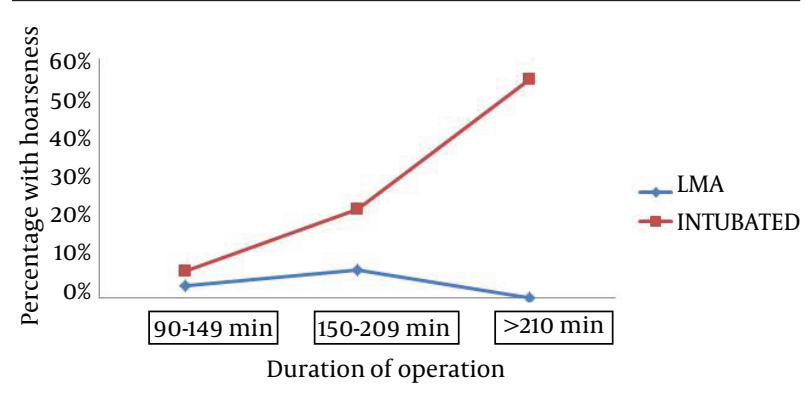

The incidence increased in patient who had longer ENT surgeries (90 to 210 minutes).

\section{Discussion}

Our study excluded ENT patients with airway anomalies, respiratory diseases and surgeries in throat and lower respiratory regions, which could influence postoperative respiratory complications. The devices we use for a single invasive procedure like intubation could be associated with different incidences of respiratory complications, like what we can see in Najafi et al. (3) study, in which sore throat and hoarseness was recorded with lesser incidences for Glide Scope than Macintosh laryngoscopy. As an invasive procedure, we expect laryngoscopy to be associated with increased risk of postoperative airway complications, but laryngoscopy for LMA placement has facilitated the procedure and also decreased the incidence of sore throat $(3,4)$. We did not want to use LMA in more than three-hour surgeries, but as the operation time is not predictable, some of the surgeries took more time and when LMAs were working properly, we decided to continue the operation with those devices.

LMA as a supraglottic device in elective ENT operations is now considered as a standard choice (1). Although the duration of operation could be a limiting factor, the use of supraglottic device for elective prolonged ENT operations has been reported since $2001(4,5)$. The traditional fear of increased risk of gastric content aspiration with LMA does not exist anymore $(5,6)$. Among different supraglottic devices (6-8), ProSeal LMA and Flexible LMA seem to be more suitable for ENT operations.

We considered sore throat as any kind of unpleasant 
sensation in the throat region like pain with or without swallowing. In some studies this symptom has been divided into different types like sore throat, dysphagia, pharyngeal dryness and even jaw pain (8-10).

Sore throat in immediate postoperative period was more common in the intubated patients (44.2\% vs. $32.9 \%$ ); but, the difference was not significant. It was more common in 15 - 29-year-old patients and its incidence decreased in older candidates. In outpatient surgery (when we expect less prolonged surgeries), the incidence of sore throat was 3\% for mask ventilation, 18\% for LMA and 45\% for endotracheal tube (1). In Grady et al. (2) study, more prolonged operation was associated with increased incidence of sore throat ( $49 \pm 14$ minutes vs. $39 \pm 14$ minutes). Najafi et al. found higher incidence of sore throat in longer surgeries (3), but most studies have not considered length of surgery as a factor in changing the incidence of postoperative respiratory complications. It seems that the overall incidences of postoperative sore throat have been 14\% -50\% for tracheal tube and 5.8\% - 34\% for LMA $(8,9)$; however, some studies have found the incidence of postoperative sore throat to be as high as $92 \%(10,11)$. Oh et al. (12) divided this symptom to sore throat and dysphagia, but the sum of their incidences for LMA (31\%) was identical to our findings. Orandi et al. recorded sore throat in 51\% of intubated females who had 110-minute surgeries (13). There are also studies that have found similar incidences of sore throat for endotracheal intubation and LMA $(13,14)$. Our study was performed in a teaching hospital and some complications that are directly related to the experience of the trainees were expected to be more common. According to this study, the risk of sore throat with LMA for prolonged ENT operation was not more than tracheal tube and in this range of time (90 to 210 minutes) the risk of sore throat for endotracheal tube and LMA did not increase with time.

Different methods have been tested for reducing the risk of postoperative sore throat after tracheal intubation or LMA placement. Using laryngoscope and introducers have lowered the sore throat incidence of LMA $(14,15)$. Ketamine gargle, application of betamethasone gel (8 7), Intra-cuff dexamethasone (16), selection of smaller tubes with smaller cuffs, avoiding the application of anesthetic spray and administration of succinylcholine are some of the different methods proposed to reduce the incidence of sore throat (1, 8-10, 15, 17-19).

Mild local irritation and edema of the vocal cords are usually the causes of hoarseness, which resolve in several postoperative days. More prolonged postoperative hoarseness could also be seen, which heralds more severe injuries. Recurrent laryngeal nerve trauma, contactulcer granuloma, laryngeal cartilage injuries and vocal cord hematoma are among other causes of postoperative hoarseness, which indicates how airway devices could potentially cause major injuries in respiratory structures.

Hoarseness was seen in $3.5 \%$ of the LMA patients and $24.4 \%$ of the intubated patients (Fisher's Exact test $=$
0.000). The incidence of hoarseness in intubated patients had a direct relationship with the length of surgery. For LMA, this correlation could not be seen. LMA has direct access to phonation structures, but the incidence of hoarseness in this group was significantly less than the intubated patients. Christensen et al. (19, 20), found that in as high as $50 \%$ of intubated patients hoarseness could be seen and this figure was about 15\% for LMA (2). According to our findings, the duration of operation was an important factor which increased the incidence of postoperative hoarseness in intubated patient.

Local irritation of respiratory mucosa by tracheal tube is the most common cause of cough. In ENT surgeries, we usually need to extubate an awake patient and bucking on the tube could exaggerate this mechanical irritation. The aspiration of secretion from the upper parts of the respiratory tract that could be seen for both tracheal tube and LMA is another cause of tracheobronchial mucosal irritation. LMA does not enter the trachea; so, mechanical irritation does not occur; it could also be an effective barrier against fluids entrance (1). The incidence of cough in the LMA patients was $1.2 \%$ and for the intubated patients it was $12.8 \%$ (Fisher's Exact test $=0.005$ ). Cough was more common among female patients (10.7\% vs. 1.5\%). Kristensen recorded cough in $18.5 \%$ of intubated patients (19, 20 ). Grady et al. (2) observed cough in $25 \%$ of intubated patients, but in $0 \%$ of patients with laryngeal mask. Our observation showed a lower incidence of cough in the LMA group than in the intubated patients. It may have also indicated that LMA produces were more effective isolation methods for the lower respiratory tract.

Shortness of breath was seen in two intubated patients, but no significant difference was observed in comparison with the LMA group. In patients who had shortness of breath, we could not find significant tachypnea or respiratory distress.

We observed a significant difference in the incidence of patients with least one respiratory complication (34\% vs. $37 \%$ ) and patients with more than one respiratory complication (2.4\% vs. $22.1 \%$ ) between the two groups (Table 2 ). If we consider this finding as a surrogate marker of respiratory system invasion, we can conclude that LMA is a less invasive device and its degree and extent of insult is less than endotracheal tube.

The aspiration of gastric content in elective patients with LMA did not occur more than in patients with tracheal tube. In most ENT surgeries, head-up position is the preferred intraoperative position. This position prevents gastric content regurgitation into the esophagus and hence into the lower respiratory tract. It also helps the diaphragm to move freely and with less pressure gradient. We did not see gastric distension in our patients, but this complication could occur in all the cases with LMA, regardless of the length of surgery. In patients with LMA, gastric distention must be monitored continuously, and if it could not be corrected by repositioning, we must change LMA to tracheal tube. 
Only in one of our patients LMA could not maintain sufficient ventilation, which was detected in the beginning of the surgery. There was no critical incidence during the intraoperative period in other cases. We found that LMA was an easy device for airway control in ENT operations longer than 90 minutes. Hoarseness and cough were less common with this device in patients with normal airway anatomy. The fixation of this device for such prolonged operations in which we have limited access to the patient's face must be done properly. In some occasional cases of FESS surgery, LMA leakage may produce fog or splashes of blood on the endoscopy lens, which could make the surgeon change the LMA to tracheal tube.

One of our main limitations in this study was the surgeons who thought LMA was unsafe and could not prevent the aspiration of the upper airway secretion, but they adapted with the device during the study progress and found it reliable.

\section{Acknowledgements}

We would like to thank Dr. Masoud Solaymani-Dodaran for his advices during this study.

\section{Authors' Contributions}

Reza Safaeian and Valiollah Hassani: study concept and design, data acquisition. Mahzad Alimian, Reza Safaeian, Valiollah Hassani, Gholamreza Movasaghi, and Hamid Reza Faiz: analysis and interpretation. Gholamreza Movasaghi: manuscript drafting. Hamid Reza Faiz: critical revision. Reza Safaeian: administrative support and study supervision.

\section{Funding/Support}

Funding was provided by Iran university of medical sciences.

\section{References}

1. Thornton JA. Anesthesia for Eye, Ear, Nose, and Throat Surgery. Br Med J. 2010;3:547.

2. Grady DM, McHardy F, Wong J, Jin F, Tong D, Chung F. Pharyngolaryngeal morbidity with the laryngeal mask airway in spontaneously breathing patients: does size matter? Anesthesiology. 2001;94(5):760-6.

3. Najafi A, Imani F, Makarem J, Khajavi MR, Etezadi F, Habibi S, et al. Postoperative sore throat after laryngoscopy with macintosh or glide scope video laryngoscope blade in normal airway patients. Anesth Pain Med. 2014;4(1):e15136.

4. Maruyama K, Nakagawa H, Imanishi H, Kitamura A, Hayashida $\mathrm{M}$. Comparison of postoperative pharyngeal morbidity using the Macintosh laryngoscope or AirWay Scope after mastectomy. J Anesth. 2011;25(5):773-6.

5. Nicholls M. ProSeal laryngeal mask airway use for prolonged middle ear surgery. BrJAnaesth. 2001;87(2):323-4.

6. Brimacombe JR, Berry A. The incidence of aspiration associated with the laryngeal mask airway: a meta-analysis of published literature. J Clin Anesth. 1995;7(4):297-305.

7. Alexiev V, Salim A, Kevin LG, Laffey JG. An observational study of the Baska(R) mask: a novel supraglottic airway. Anaesthesia. 2012;67(6):640-5.

8. Joe HB, Kim DH, Chae YJ, Kim JY, Kang M, Park KS. The effect of cuff pressure on postoperative sore throat after Cobra perilaryngeal airway. J Anesth. 2012;26(2):225-9.

9. McHardy FE, Chung F. Postoperative sore throat: cause, prevention and treatment. Anaesthesia.1999;54(5):444-53.

10. William A, Chambers NA, Erb TO, von Ungern-Sternberg BS. Incidence of sore throat in children following use of flexible laryngeal mask airways - impact of an introducer device. Paediatr Anaesth. 2010;20(9):839-43.

11. Shaaban AR, Kamal SM. Comparison between betamethasone gel applied over endotracheal tube and ketamine gargle for attenuating postoperative sore throat, cough and hoarseness of voice. Middle East J Anaesthesiol. 2012;21(4):513-9.

12. Oh SK, Lim BG, Kim H, Lim SH. Comparison of the clinical effectiveness between the streamlined liner of pharyngeal airway (SLIPA) and the laryngeal mask airway by novice personnel. Korean J Anesthesiol. 2012;63(2):136-41.

13. Orandi A, Orandi A, Najafi A, Hajimohammadi F, Soleimani S, Zahabi S. Post-intubation sore throat and menstruation cycles. Anesth Pain Med. 2013;3(2):243-9.

14. Rieger A, Brunne B, Hass I, Brummer G, Spies C, Striebel HW, et al. Laryngo-pharyngeal complaints following laryngeal mask airway and endotracheal intubation. J Clin Anesth. 1997;9(1):42-7.

15. Griffiths JD, Nguyen M, Lau H, Grant S, Williams DI. A prospective randomised comparison of the LMA ProSeal versus endotracheal tube on the severity of postoperative pain following gynaecological laparoscopy. Anaesth Intensive Care. 2013;41(1):46-50.

16. Choo CY, Koay CK, Yoong CS. A randomised controlled trial comparing two insertion techniques for the Laryngeal Mask Airway Flexible in patients undergoing dental surgery. Anaesthesia. 2012;67(9):986-90.

17. Solatpour F, Teymourian H, Mohajerani SA, Hoseinzadegan Shirazi F, Lotfollah Zadeh S, Baikpour M, et al. Comparison of the incidence of sore throat after rapid sequence intubation with succinylcholine and cisatracurium. Anesth Pain Med. 2014;4(3):e20030.

18. Henderson J, Miller RD. Miller's anesthesi.Philadelphia: Elsevier Churchill Livingston; 2010.

19. Rafiei MR, Arianpour N, Rezvani M, Ebrahimi A. Effects of intracuff dexamethasone on post-extubation reactions. J Res Med Sci. 2012;17(4):338-43.

20. Christensen AM, Willemoes-Larsen H, Lundby L, Jakobsen KB. Postoperative throat complaints after tracheal intubation. $\mathrm{Br} J$ Anaesth.1994;73(6):786-7. 